\title{
Disseminated Bacille Calmette-Guérin Infection in Immunodeficient Infants: Report of Two Cases
}

\author{
Ahmed Ben Hadj Hassine, ${ }^{1,2,}$ Manel Marzouk, ${ }^{1}$ Hichem Bargui, ${ }^{1,2}$ Miniar Tfifha, ${ }^{3}$ Mohamed Dhaou, ${ }^{1}$ \\ and Jalel Boukadida ${ }^{1}$ \\ ${ }^{1}$ Laboratory of Microbiology and Immunology, University Hospital Farhat Hached, Sousse, Tunisia \\ ${ }^{2}$ Faculty of Pharmacy of Monastir, University of Monastir, Monastir, Tunisia \\ ${ }^{3}$ Service of Pediatrics Diseases, University Hospital Sahloul, Sousse, Tunisia \\ "Corresponding author: Ahmed Ben Hadj Hassine, Laboratory of Microbiology and Immunology, University Hospital Farhat Hached, Sousse, Tunisia. E-mail: \\ benhadjhassine.ahmed@yahoo.fr
}

Received 2016 March 06; Revised 2016 August 21; Accepted 2016 September 05.

\begin{abstract}
Introduction: The Bacille Calmette-Guérin (BCG), a live attenuated Mycobacterium bovis vaccine, is administered to all the newborns in Tunisia in order to prevent Tuberculosis (TB). Complications of this vaccine are uncommon. However, it poses a risk for children with unknown immunodeficiency.

Case Presentation: We report on disseminated BCG disease in two infants, respectively, with severe combined immunodeficiency and human immunodeficiency virus (HIV). Evolution was fatal for both, despite adequate anti-tuberculosis treatment.

Conclusions: Molecular methods are available to respond to the urgent need for rapid and specific diagnosis of local/regional or systemic BCG disease, using available commercial kits GenoType $\circledR^{\circledR}$ MTBC and GenoType $®$ MTBDRplus. These tests allow prevention of inoculation of live vaccines such as BCG among the next siblings until appropriate screening tests exclude primary or secondary immunodeficiency syndromes.
\end{abstract}

Keywords: Bacille Calmette-Guérin Vaccine, Mycobacterium bovis, Molecular Diagnostic Techniques, Immunologic Deficiency Syndromes

\section{Introduction}

The Bacille Calmette-Guerin (BCG) vaccine, composed of a live, attenuated Mycobacterium bovis strain, is administered to almost 100 million children annually and remains the only licensed vaccine for tuberculosis prevention (1). Bacille Calmette-Guerin induces strong T-helper type 1 (Th1) responses, even in neonates (1) and protects against severe forms of childhood tuberculosis, such as tuberculous meningitis and miliary tuberculosis in immunocompetent children (2). In regions of high tuberculosis endemicity, such as Tunisia, BCG vaccine is administered soon after birth. Although it is considered safe in general, there are several complications, ranging from local/regional BCG disease to disseminated BCG disease. However, disseminated BCG disease, which is associated with a high mortality rate, is very rare. This BCG complication may suggest an underlying immunodeficiency (38). In Tunisia, BCG substrain Pasteur vaccine, is administered to all the children at birth to prevent TB, without any immunity status information. In addition, conventional biochemical mycobacteria identification tests are complex and time consuming, causing adverse consequences in the management of patients. To overcome this, molecular methods are used, for rapid and effective diagnostic tools.

Here, we report on two infants, who developed disseminated BCG disease after BCG vaccination. Bacteriological diagnosis was confirmed by molecular methods. When definite diagnosis was made, immunological evaluation was done, which revealed, severe combined immunodeficiency disease and infection with Human Immunodeficiency Virus (HIV), respectively.

\section{Case Presentation}

The first case is a four-month-old male, with prolonged fever lasting for one month before admission; his illness did not respond to treatment by prescribing antibiotics. The infant was hospitalized at the pediatric department on the $26^{\text {th }}$ of June 2015 (day 0 = day of admission). At admission, physical examination revealed hepatomegaly and splenomegaly. The infectious investigations showed high levels of C-Reactive-Protein and leukocytosis (14000 $/ \mathrm{mm}^{3}$ ). Cyto-bacteriological urine test and culture were negative. At day +3 , blood cultures were positive for Klebsiella pneumoniae producing beta lactamase extended spectrum. Despite antibiotic therapy adapted to the antibiogram's results, and negativity of blood cultures, the child 
still remained febrile. At day +6 , ultrasonography revealed a multi-nodular diffuse liver damage and X-ray showed a lytic bone lesion affecting long and flat bones. The diagnostics of neuroblastoma metastasis, histiocytosis $\mathrm{X}$ and mycobacteriosis were discussed. A bone marrow biopsy was performed on day +7 . Mycobacteriology examination was performed on the same day, including microscopy by Ziehl-Neelsen technique, and showed acid-fast bacilli with a high bacterial load (three crosses). Tuberculosis or BCG infection diagnosis was made, and the patient was then started on anti-tuberculosis quadritherapy: isoniazide, rifampicin, streptomycin and ethambutol. This diagnosis led to realization of immunological tests, which revealed a severe combined immunodeficiency (SCID) in this case; patient characteristics are summarized in Table 1.

The second case was a six-month old female, with prolonged fever and pulmonary symptoms (persistent cough and dyspnea) lasting for three weeks before admission. His illness did not respond to treatment by prescribing antibiotics. On the 18th of June 2015, the infant was hospitalized in the pediatric department (day 0 = day of admission). Cyto-bacteriological urine and blood cultures were negative. On day +2 , gastric aspiration was positive on acid-fast bacilli microscopy by Ziehl-Neelsen technique ( 1 cross). Tuberculosis or BCG infection diagnosis was made and the patient was then started on anti-tuberculosis quadritherapy: isoniazide, rifampicin, streptomycin and ethambutol. Immunological investigations and viral serology, performed on day +3 , revealed a positive serology to HIV in this infant. Her mother was also HIV positive; the patient characteristics are summarized in Table 1.

In the two cases, cultures of Mycobacteria on Lowenstein Jensen medium became positive on day +21 for the bone marrow biopsy and day +28 for the gastric aspiration. Strains identification was performed from colonies by genotypic method using the kit GenoType $®$ MTBC (Hain Lifescience, Germany). The anti-tuberculosis susceptibility was performed by the kit GenoType $®$ MTBDRplus (Hain Lifescience, Germany). The two kits were used according to the protocol recommended by the manufacturer. Both techniques are based on DNA • STRIP® assays, which are based on a multiplex polymerase chain reaction (PCR) in combination with reverse hybridization. The GenoType $®$ MTBC assay is based on M. tuberculosis complex-specific 23s ribosomal DNA fragments, gyrB DNA sequence polymorphisms and the RD1 deletion region of $M$. bovis BCG. The Genotype MTBDRplus assay, used for anti-tuberculosis susceptibility, is based on rpoB, katG and inhA mutations detection to determinate isoniazid and rifampicin resistance. DNA extraction was performed by the thermal method $\left(45\right.$ minutes at $\left.95^{\circ} \mathrm{C}\right)$ from discount colonies in suspension. Briefly, $5 \mu \mathrm{L}$ of extracted DNA was added to $20 \mu \mathrm{L}$ of commercialized master mix in a PCR tube, then amplified according to the supplier's instructions: five minutes at $95^{\circ} \mathrm{C}$, followed by 10 cycles $\left(30\right.$ seconds, $95^{\circ} \mathrm{C}$ and two minutes, $58^{\circ} \mathrm{C}$ ), and 20 cycles $\left(25\right.$ seconds, $95^{\circ} \mathrm{C} ; 40 \mathrm{~s} \mathrm{~min}$, $53^{\circ} \mathrm{C}$ and 40 seconds, $70^{\circ} \mathrm{C}$ ) ended by final elongation for eight minutes at $70^{\circ} \mathrm{C}$ ).

Both strains were identified: M. bovis BCG, based on the hybridization profile indicating the specific band of the tuberculosis complex (band 4) associated with specific bands of the species BCG (band 7, 9, 10 and 13) (Figure 1). The two strains showed wild genotype rpoB, katG and inhA and therefore were sensitive to rifampicin and isoniazid (Figure 2).

Figure 1. Identification of the Isolated Strain by GenoType $₫$ MTBC kit (Hain Lifescience, Germany)

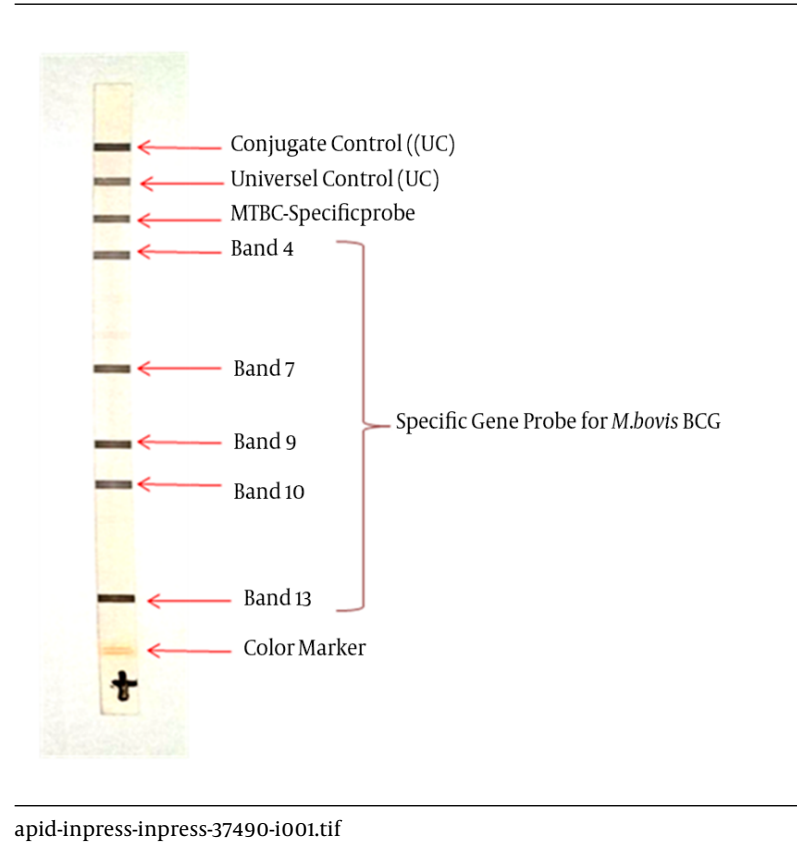

Despite the two children receiving adequate antituberculosis treatment, they died on day +52 and +58 respectively, due to severe disseminated BCG infection.

\section{Discussion}

The BCG vaccine is a widely practiced vaccine, which is useful for prophylaxis against tuberculosis. Disseminated BCG infection is one of the most important complications of this vaccine and can be seen in patients with an underlying immunodeficiency (9). Although immunological and HIV statutes are not known at birth, routine BCG vaccination continues in most countries, including Tunisia.

Although the current study emphasized on the diagnostic ability of molecular tests in accurate diagnosis of 
Table 1. Cases Descriptions

\begin{tabular}{|c|c|c|}
\hline & Case 1 & Case 2 \\
\hline Gender & Male & Female \\
\hline Age (month) & 4 & 6 \\
\hline Reasons of hospitalization & Prolonged fever of one month & $\begin{array}{l}\text { Prolonged fever and pulmonary symptoms (persistent } \\
\text { cough, mild dyspnea) of } 3 \text { weeks }\end{array}$ \\
\hline \multicolumn{3}{|l|}{ Realized clinical exams and results } \\
\hline Physical examination & Hepatomegaly and splenomegaly & Not available \\
\hline Infectious exams & $\begin{array}{l}\text { High levels of C-Reactive-Protein and leukocytosis (14000 } \\
\left(\mathrm{mm}^{3}\right)\end{array}$ & HIV positive (for case and her mother) \\
\hline Cytobacteriological urine test & Negative & Negative \\
\hline Blood cultures & $\begin{array}{l}\text { Positive (K. pneumoniae with } \beta \text {-lactamase extended } \\
\text { spectrum) }\end{array}$ & Negative \\
\hline \multicolumn{3}{|l|}{ Radiological investigation } \\
\hline Ultrasonography & Multi-nodular with diffuse liver damage & Not available \\
\hline $\mathrm{X}$ ray & Lytic bone lesions (long and flat bones) & Not available \\
\hline \multicolumn{3}{|l|}{ Mycobacteriology } \\
\hline Ziehl-Neelsen microscopy & Positive for bone morrow ponction (high bacterial load) & Positive for gastric aspiration (low bacterial load) \\
\hline Culture on LJ medium & Positive (on day 21) & Positive (on day 28) \\
\hline Diagnostics & (SCID) & HIV \\
\hline Established treatment & $\begin{array}{l}\text { Anti-tuberculosis quadritherapy: isoniazide, rifampicin, } \\
\text { streptomycin and ethambutol }\end{array}$ & $\begin{array}{l}\text { Anti-tuberculosis quadritherapy: isoniazide, rifampicin, } \\
\text { streptomycin and ethambutol }\end{array}$ \\
\hline Admission date & 26 June 2015 & 18 June 2015 \\
\hline Duration of admission (day) & 52 & 58 \\
\hline Death date & 16 August 2015 & 14 August 2015 \\
\hline
\end{tabular}

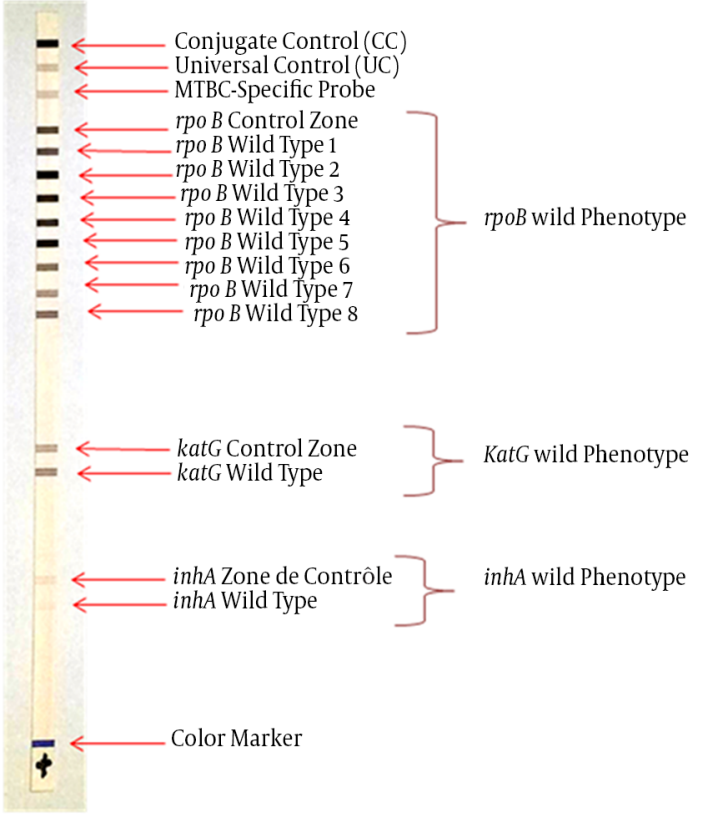

Figure 2. Anti-Tuberculosis Susceptibility by GenoType $₫$ MTBDRplus kit (Hain Lifescience, Germany) disseminated BCG disease yet, in such cases with compatible clinical signs and symptoms, who are suspected of having immunodeficiency syndromes, a positive AFB smear in BMA is sufficient to start specific anti-TB treatment and in such situation, molecular tests only act as a confirmatory modality. Treatment should not be delayed till the results of PCR or cultures are available.

The diagnosis of disseminated BCG infection, often unrecognized or late, generally reveals severe immunodeficiency diseases in infants $(3,7,9)$. It is reported that disseminated BCG disease is one of the most common causes of death in patients with primary immunodeficiency diseases, particularly in those with SCID (10) and in HIVinfected infants in the absence of combined antiretroviral therapy $(11,12)$. This poor prognosis is due to clinical and bacteriological conventional diagnostic delay (10). To overcome this, genotypic methods are used to accelerate the bacteriological diagnosis, to improve the therapeutic management and to prevent the BCG vaccine in the next siblings, asking an accurate diagnosis. These methods are based on reverse hybridization techniques for the identification of Mycobacteria species and anti-tuberculosis susceptibility from positive cultures on solid or liquid media $(13,14)$. However, a few studies have reported the identification of M. bovis BCG and its anti-tuberculosis susceptibility by these kits (15). This could be explained by the 
fact that disseminated BCG disease is found in developing countries, where, molecular techniques, are of limited use due to lack of resources (16), which explains why only a few studies report the use of a molecular method (PCR) on tissue specimens with good diagnostic yield, especially on bone marrow and liver specimens (17). The use of conventional techniques to identify $M$. bovis BCG is fastidious and slow, so diagnostic of BCG infections seems difficult in these countries (3). The second advantage of molecular techniques is that they provide rapid results of susceptibility of $M$. bovis BCG strain to anti-tuberculosis simultaneously with diagnosis, improving therapeutic management (15).

Despite fast local/regional BCG disease diagnosis and rapid reporting of sensitivity to anti-tuberculosis, the evolution was fatal for both infants. However, we were able to diagnose the species M. bovis BCG using molecular techniques and label the disseminated BCG infection as such, allowing us to reveal, respectively, SCID and HIV infection.

Therefore, for the next siblings, inoculation of live vaccines such as BCG would be postponed for a few months, until appropriate screening tests exclude this diagnosis; vaccination should then be performed in those with an intact immune system.

\section{Footnotes}

Authors' Contribution: All authors are responsible for reported research and have participated in the concept and design, analysis and interpretation of data, drafting and revising of the manuscript, and have approved this manuscript as submitted.

Funding/Support: This work was financed by the MESRS UR12SP34.

\section{References}

1. Marchant A, Goetghebuer T, Ota MO, Wolfe I, Ceesay SJ, De Groote D, et al. Newborns develop a Th1-type immune response to Mycobacterium bovis bacillus Calmette-Guerin vaccination. J Immunol. 1999;163(4):2249-55. [PubMed:10438968].

2. Trunz BB, Fine P, Dye C. Effect of BCG vaccination on childhood tuberculous meningitis and miliary tuberculosis worldwide: a meta-analysis and assessment of cost-effectiveness. Lancet. 2006;367(9517):1173-80. doi:10.1016/S0140-6736(06)68507-3.

3. Casanova JL, Jouanguy E, Lamhamedi S, Blanche S, Fischer A. Immunological conditions of children with BCG disseminated infection. Lancet. 1995;346(8974):581. [PubMed: 7658805].
4. Cohn DL. Use of the bacille Calmette-Guerin vaccination for the prevention of tuberculosis: renewed interest in an old vaccine. Am J Med Sci. 1997;313(6):372-6. [PubMed: 9186153].

5. Gonzalez B, Moreno S, Burdach R, Valenzuela MT, Henriquez A, Ramos MI, et al. Clinical presentation of Bacillus Calmette-Guerin infections in patients with immunodeficiency syndromes. Pediatr Infect Dis J. 1989;8(4):201-6. [PubMed: 2654859].

6. Casanova JL, Blanche S, Emile JF, Jouanguy E, Lamhamedi S, Altare F, et al. Idiopathic disseminated bacillus Calmette-Guerin infection: a French national retrospective study. Pediatrics. 1996;98(4 Pt 1):774-8. [PubMed: 8885960].

7. Afshar Paiman S, Siadati A, Mamishi S, Tabatabaie P, Khotaee G. Disseminated Mycobacterium bovis infection after BCG vaccination. Iran J Allergy Asthma Immunol. 2006;5(3):133-7. [PubMed: 17237565].

8. Deeks SL, Clark M, Scheifele DW, Law BJ, Dawar M, Ahmadipour N, et al. Serious adverse events associated with bacille Calmette-Guerin vaccine in Canada. Pediatr Infect Dis J. 2005;24(6):538-41. [PubMed: 15933565].

9. Sadeghi-Shabestari M, Rezaei N. Disseminated bacille CalmetteGuerin in Iranian children with severe combined immunodeficiency. Int J Infect Dis. 2009;13(6):420-3. doi:10.1016/j.ijid.2009.02.008. [PubMed: 19403320].

10. Rezaei N, Aghamohammadi A, Moin M, Pourpak Z, Movahedi M, Gharagozlou M, et al. Frequency and clinical manifestations of patients with primary immunodeficiency disorders in Iran: update from the Iranian Primary Immunodeficiency Registry. J Clin Immunol. 2006;26(6):519-32. doi: 10.1007/s10875-006-9047-x. [PubMed: $17024564]$.

11. Hesseling AC, Rabie H, Marais BJ, Manders M, Lips M, Schaaf HS, et al. Bacille Calmette-Guerin vaccine-induced disease in HIV-infected and HIV-uninfected children. Clin Infect Dis. 2006;42(4):548-58. doi: 10.1086/499953. [PubMed: 16421800].

12. Rabie H, Violari A, Duong T, Madhi SA, Josipovic D, Innes S, et al. Early antiretroviral treatment reduces risk of bacille Calmette-Guerin immune reconstitution adenitis. Int J Tuberc Lung Dis. 2011;15(9):1194200. doi: 10.5588/ijtld.10.0721. [PubMed: 21943845] i.

13. Barnard M, Gey van Pittius NC, van Helden PD, Bosman M, Coetzee G Warren RM. The diagnostic performance of the GenoType MTBDRplus version 2 line probe assay is equivalent to that of the Xpert MTB/RIF assay. J Clin Microbiol. 2012;50(11):3712-6. doi: 10.1128/JCM.01958-12. [PubMed: 22972826].

14. Hillemann D, Rusch-Gerdes S, Richter E. Evaluation of the GenoType MTBDRplus assay for rifampin and isoniazid susceptibility testing of Mycobacterium tuberculosis strains and clinical specimens. J Clin Microbiol. 2007;45(8):2635-40. doi: 10.1128/JCM.00521-07. [PubMed: 17537937].

15. Yadav RN, Singh BK, Sharma SK, Sharma R, Soneja M, Sreenivas V, et al. Comparative evaluation of GenoType MTBDRplus line probe assay with solid culture method in early diagnosis of multidrug resistant tuberculosis (MDR-TB) at a tertiary care centre in India. PLoS One. 2013;8(9):72036. doi: 10.1371/journal.pone.0072036. [PubMed: 24039735].

16. Ying W, Sun J, Liu D, Hui X, Yu Y, Wang J, et al. Clinical characteristics and immunogenetics of BCGosis/BCGitis in Chinese children: a 6 year follow-up study. PLoS One. 2014;9(4):94485. doi: 10.1371/journal.pone.0094485. [PubMed: 24722620].

17. Aelami MH, Alborzi A, Pouladfar G, Geramizadeh B, Pourabbas B, Mardaneh J. Post-Vaccination Disseminated Bacillus Calmette Guerin Infection Among Children in Southern Iran. Jundishapur J Microbiol. 2015;8(11):25663. doi: 10.5812/jjm.25663. [PubMed: 26862381]. 